\title{
Are there monopoles in the quark-gluon plasma?
}

\author{
Adith Ramamurti, ${ }^{*}$ Edward Shuryak, ${ }^{\dagger}$ and Ismail Zahed ${ }^{\ddagger}$ \\ Department of Physics and Astronomy, Stony Brook University, Stony Brook, New York 11794, USA
}

(Received 18 April 2018; published 28 June 2018)

\begin{abstract}
Monopolelike objects have been identified in multiple lattice studies, and there is now a significant amount of literature on their importance in phenomenology. Some analytic indications of their role, however, are still missing. The 't Hooft-Polyakov monopoles, originally derived in the Georgi-Glashow model, are an important dynamical ingredient in theories with extended supersymmetry $\mathcal{N}=2,4$, and help explain the issues related with electric-magnetic duality. There is no such solution in QCD-like theories without scalar fields. However, all of these theories have instantons and their finite- $T$ constituents known as instanton-dyons (or instanton-monopoles). The latter leads to semiclassical partition functions, which for $\mathcal{N}=2,4$ theories were shown to be identical ("Poisson dual") to the partition function for monopoles. We show how, in a pure gauge theory, the semiclassical instanton-based partition function can also be Poissontransformed into a partition function, interpreted as the one of moving and rotating monopoles.
\end{abstract}

DOI: 10.1103/PhysRevD.97.114028

\section{INTRODUCTION}

The possible existence of magnetic monopoles in electrodynamics fascinated leading physicists in the 19th century. With the development of quantum mechanics, Dirac [1] related the existence of monopoles with the electric charge quantization. However, QED monopoles were never found.

Classical solitons with magnetic charge were found by 't Hooft [2] and Polyakov [3] in the Georgi-Glashow model. Such monopoles exist and play an important role in other theories with an adjoint scalar field, notably in theories with extended supersymmetry $\mathcal{N}=2$, 4. Their presence and properties have significantly advanced our understanding of the electric-magnetic duality and its relation to the renormalization group (RG) flow. In the $\mathcal{N}=2$ case, there is a gradual transition from an electric theory at weak coupling to a magnetic theory at strong coupling [4]. In the $\mathcal{N}=4$ case, monopoles dressed by bound fermions were shown to create an $\mathcal{N}=4$ multiplet of fields, making the electric and magnetic theories the same, up to a coupling. This implies that the beta function of $g$ and $1 / g$ must be the same, therefore just zero, explaining why this theory must be conformal.

\footnotetext{
*adith.ramamurti@stonybrook.edu †edward.shuryak@stonybrook.edu

*ismail.zahed@stonybrook.edu
}

Published by the American Physical Society under the terms of the Creative Commons Attribution 4.0 International license. Further distribution of this work must maintain attribution to the author(s) and the published article's title, journal citation, and DOI. Funded by SCOAP.
In QCD-like theories without scalars, e.g., pure gauge theories or $\mathcal{N}=1 \mathrm{SYM}$, there are no such monopole solutions. Despite this, Nambu [5], 't Hooft [6], and Mandelstam [7] proposed the "dual superconductor" model of the electric color confinement. In this model, the Bose-Einstein condensation (BEC) of monopoles at $T \leq T_{c}$ expels electric fields from the vacuum into confining flux tubes.

In lattice studies of gauge theories, monopoles have been identified, and their locations and paths were correlated with gauge-invariant observables, such as the action and square of the magnetic field [8]. The monopoles were found to create a magnetic current around the electric flux tube $[9,10]$. In Landau gauge, while monopole-type singularities themselves are not present, the physical properties that they source are still present and gauge-invariant [11]. The motion and correlations of the monopoles were shown to be as expected for a Coulomb plasma [12-14], the deconfinement critical temperature $T_{c}$ does coincide accurately with that of monopole BEC transition $[13,15,16]$, and the BEC transition has been shown to be gauge independent [17-19].

The "magnetic scenario" for quark-gluon plasma (QGP) $[14,20,21]$ assumes the presence of noncondensed monopoles as another kind of quasiparticles. Due to electric confinement at $T<T_{c}$, quarks and gluons have vanishing densities. More specifically, the density of quarks, as the derivative over the chemical potential $\mu_{b}$ (at $\mu_{b}=0$ in this paper), is directly observed on the lattice. Near $T_{c}$ it is proportional to the expectation value of the Polyakov line $\langle P(T)\rangle$. The gluon density is assumed to be proportional to its square.) In contrast to that the monopoles do not have color electric charge and their density has a peak near $T_{c}$. 


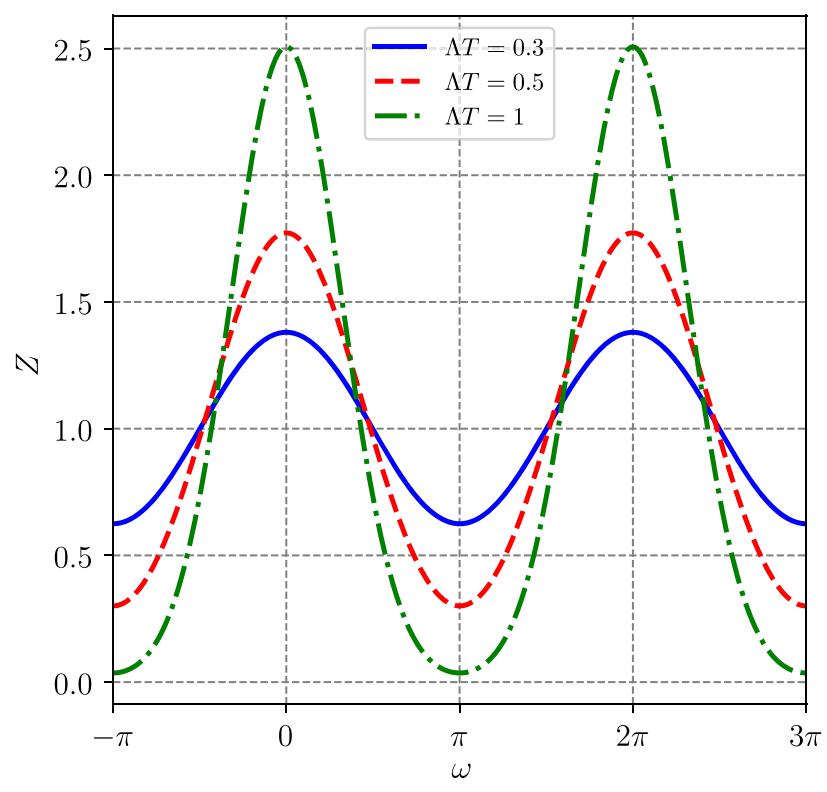

FIG. 1. The partition function $Z$ of the rotator as a function of the external Aharonov-Bohm phase $\omega$ (two periods are shown to emphasize its periodicity). The (blue) solid, (red) dashed and (green) dash-dotted curves are for $\Lambda T=0.3,0.5,1$.

(The monopole density can either be directly deduced from lattice simulation, or deduced from the known total free energy, in the assumption that these three components reproduce it (see e.g., Fig. 1 of our previous paper [22] in which all components are shown as a function of $T$ ).

Monopole-gluon and monopole-quark scattering were shown to play a significant role in kinetic properties of the QGP, such as the shear viscosity $\eta[23]$ and the jet quenching parameter $\hat{q}[22,24,25]$. The noncondensed monopoles should also lead to electric flux tubes at $T>T_{c}$ [21], which were recently observed on the lattice [26]. Thus, there is a growing amount of phenomenological evidence suggesting magnetic monopoles do exist, not only as a confining condensate at $T \leq T_{c}$, but also as noncondensed quasiparticles at $T>T_{c}$. While the central role of monopoles in the confinement-deconfinement transition was recognized long ago, their relation to another important nonperturbative aspect of QCD-like theories, chiral symmetry breaking, has attracted much less attention prior to our recent paper [27], in which we have demonstrated how the quark condensate is formed as $T \rightarrow T_{c}$.

Nevertheless, this phenomenological evidence does not convince many theorists, who would rather have an analytic argument not relying on lattice numerics or heavy-ion phenomenology. One such argument will be provided by this paper. It is still indirect, in the sense that we do not have a microscopic description of these monopoles in terms of the gauge fields. We do, however, derive the corresponding partition function, based on a transformed semiclassical partition function.
The semiclassical description of the vacuum of gauge theories is based on the instanton solution [28]. At finite temperatures, however, the four-dimensional instanton solution has been shown to dissolve into instanton constituents, known as instanton-dyons (or instanton-monopoles) [29-31]. Studies of the ensembles of instanton-dyons have explained the deconfinement and chiral symmetry restoration transitions both numerically [32,33] and using a mean-field analysis [34,35]. For a recent short review, see Ref. [36].

The construction of the instanton-dyons starts from the same 't Hooft-Polyakov monopole, but with the fourth component of the gauge field $A_{4}$ acting as the scalar adjoint "Higgs" field. However, these objects are pseudo-particles and not particles, existing only in the Euclidean formulation of the theory for which $A_{4}$ is real. Therefore, while instanton-dyons do lead to successful semiclassical applications, their usage for phenomenological applications is severely limited. Another obstacle to their development, perhaps even more important for many, is that their physical meaning remains rather obscure. In this paper, we argue that it should not be so, and that the instanton-dyon gauge field configurations are nothing else but quantum paths of moving and rotating monopoles.

A gradual understanding of this statement began some time ago, but remained rather unnoticed by the larger community. One reason for that was the setting in which it was shown, which was based on extended supersymmetry. Only in these cases was one able to derive reliably both partition functions-in terms of monopoles and instanton-dyons-and show them to be equal [37-39]. Furthermore, they were not summed up to an analytic answer, but shown instead to be related by the so-called "Poisson duality."

Since this concept it also not widely known, Sec. II contains a pedagogical section, which discusses a much simpler toy model of a rotator-a particle on a circle-at finite temperature. We also obtain two expressions for its partition function, one based on its excited states and one based on "winding paths" in Euclidean time. In this model, one can derive the analytic solution for both sums and directly see that they are the same.

In Sec. III, we turn to theories with extended supersymmetry. This section is a brief pedagogical review of the works of Dorey and collaborators, and shows how the Poisson duality works in this case-almost identically to the rotator model.

In Sec. IV, we turn to pure gauge theories at finite temperature, using as above its simplest version with $\mathrm{SU}(2)$ color. We will explicitly derive the $n$-winding gauge configurations, periodic on the Matsubara circle, and the corresponding semi-classical partition function. We then Poisson-transform it into another form, the one we argue is counting occupations of the excited states of moving/ rotating monopoles. 


\section{QUANTUM ROTATOR AT FINITE T AND ITS DUAL DESCRIPTIONS}

A quantum rotator is a particle moving on a circle. Its location is defined by the angle $\alpha \in[0,2 \pi]$ and its action is defined by kinetic and topological parts

$$
S=\oint \mathrm{d} t \frac{\Lambda}{2} \dot{\alpha}^{2}+S_{\mathrm{top}}(\omega)
$$

where $\dot{\alpha}=\mathrm{d} \alpha / \mathrm{d} t$, and $\Lambda=m R^{2}$ is the corresponding moment of inertia for rotation. (It can be set to unity by appropriately selecting units, but for the purposes of this paper, we keep it.)

The topological part $S_{\text {top }} \sim \int \mathrm{d} t \dot{\alpha}(t)$ does not lead to any "force"- there is no contribution to the classical equation of motion-but it provides an extra phase factor in the quantum partition function. The phenomenon was introduced by Aharonov and Bohm in a celebrated paper [40] and is well known. We remind the reader that this phase is an external parameter which can be induced by a solenoid in extra dimensions, provided the rotating particle is charged and the time derivative is generalized to the long gauge-invariant derivative including the $A_{4}$ field.

The quantum mechanical spectrum of states is immediately obtained via quantization of the angular momentum $l$ and the partition function at temperature $T$ is

$$
Z_{1}=\sum_{l=-\infty}^{\infty} \exp \left(-\frac{l^{2}}{2 \Lambda T}+i l \omega\right)
$$

where, for convenience, we normalized the AharonovBohm contribution to a phase $\omega$. Since the angular momentum $l$ is integer-valued, each term in $Z_{1}$ is a periodic function of this phase, with a natural $2 \pi$ period. Note also that positive and negative $l$ cancel the imaginary part, so $Z_{1}$ is real. Finally, this sum is best convergent at small temperature $T$, where only a few states close to the ground state with $l=0$ need to be included.

In the dual approach, finite temperature is introduced via the standard Euclidean Matsubara time defined on another circle $\tau \in[0, \beta \equiv 1 / T]$. The path integral which leads to the partition function needs to be done over the periodic paths, $\alpha(0)=\alpha(\beta)$, so one may say that the Euclidean theory is a particle on a double torus.

Classes of paths which make a different number $n$ of rotations around the original circle can be defined as "straight" classical periodic paths

$$
\alpha_{n}(\tau)=2 \pi n \frac{\tau}{\beta},
$$

plus small fluctuations around them. Carrying out a Gaussian integral over them leads to the following partition function,

$$
Z_{2}=\sum_{n=-\infty}^{\infty} \sqrt{2 \pi \Lambda T} \exp \left(-\frac{T \Lambda}{2}(2 \pi n-\omega)^{2}\right)
$$

The key point here is that these quantum numbers, $l$ used for $Z_{1}$ and $n$ for $Z_{2}$, are very different in nature. In $Z_{1}$, each term of the sum is periodic in $\omega$, while in $Z_{2}$, this property is recovered only after summation over $n$. The temperature $T$ in $Z_{2}$ happens to be in an unusual place, in the numerator of the exponent, so this sum converges best at high temperature, unlike the sum in $Z_{1}$. Indeed, at high $T$ the Matsubara circle becomes small and the path integral is dominated by paths with small number of windings.

In spite of such differences, both expressions are in fact the same! In this toy model, it is not difficult to do the sums numerically and plot the results. Furthermore, one can also derive the analytic expressions, expressible in terms of the elliptic theta function of the third kind

$$
Z_{1}=Z_{2}=\theta_{3}\left(-\frac{\omega}{2}, \exp \left(-\frac{1}{2 \Lambda T}\right)\right),
$$

which is plotted in Fig. 1 for few values of the temperature $T$.

Mathematically, the identity of the two sums can be traced to the fact that our path integral is defined on two circles, or, equivalently, a $2 \mathrm{~d}$ torus, and the circles can be interchanged. In string theory, such relations are known as T-duality. In practice, these are the low-temperature and the high-temperature approximations, often used without noticing the exact summation and duality.

Even if one is not able to identify the sums as the same elliptic function, the equality can be seen from the observation that the sum $Z_{1}$ is the discrete Fourier transform of the Gaussian, which is known to be the "periodic Gaussian" appearing in $Z_{2}$. One can further recognize that the identity of the two sums is just a particular case of a more general relation known in mathematics as the Poisson summation formula, valid not only for a Gaussian but for arbitrary functions. For reference, let us mention here one particular version [41],

$$
\sum_{n=-\infty}^{\infty} f(\omega+n P)=\sum_{l=-\infty}^{\infty} \frac{1}{P} \tilde{f}\left(\frac{l}{P}\right) e^{i 2 \pi l \omega / P},
$$

where $f(x)$ is some function, $\tilde{f}$ is its Fourier transform, and $P$ is the period of both sums as a function of the "phase" $\omega$.

\section{SEMICLASSICAL THEORY AND MONOPOLES IN THEORIES WITH EXTENDED SUPERSYMMETRY}

\section{A. The setting}

The following discussion concerns a Euclidean theory defined on $R^{3} \times S^{1}$. In this section, unlike in the following one, all of the fields, including the fermions, have periodic 
boundary conditions on $S^{1}$, and therefore supersymmetry is not broken.

We study the weak coupling $g \ll 1$ scenario, which makes the instantons and their constituents-as well as the monopoles with actions/masses $O\left(1 / g^{2}\right)$ - heavy enough to trust the dilute gas approximation. This lets us focus on a single object and avoid finite-density (many-body) complications. In the $\mathcal{N}=4$ theory, the charge does not run and $g$ is simply an input parameter. In the $\mathcal{N}=2$ theory, however, the coupling does run, and one needs to select the circumference of the circle $\beta$ to be small enough such that the corresponding frequencies $\sim 2 \pi / \beta$ are large enough to ensure weak coupling.

Compactification of one coordinate to the circle is needed to introduce "holonomies," gauge invariant integrals over the circle $\oint \mathrm{d} x_{\mu} A^{\mu}, \oint \mathrm{d} x_{\mu} C^{\mu}$ of the electric and magnetic potentials, respectively. Their values can have nonzero expectation values, which can be viewed as external parameters given by Aharonov-Bohm fluxes through the circle induced by fields in extra dimensions. These holonomies will play important role in what follows. Dorey et al. [42] call these external parameters $\omega$ and $\sigma$, respectively.

Finally, in order to make the discussion simpler, one assumes the minimal non-Abelian color group $\mathrm{SU}\left(N_{c}\right)$ with the number of colors $N_{c}=2$. This group has only one single diagonal generator $\tau^{3}$, breaking the color group $\mathrm{SU}(2) \rightarrow \mathrm{U}(1)$.

The theories with extended supersymmetry $\mathcal{N}=2,4$ have one and six adjoint scalar fields, respectively. Recall that these two theories also have, respectively, 2 and 4 fermions, so that the balance between bosonic and fermionic degrees of freedom is perfect. For simplicity, all vacuum expectation values (VEV) of the scalars, as well as both holonomies are assumed to be in this diagonal direction, so the scalar VEVs and $\omega$ and $\sigma$ are singlevalued parameters without indices. In the general group $\mathrm{SU}\left(N_{c}\right)$, the number of diagonal directions is the Abelian subgroup, and thus the number of parameters is $N_{c}-1$.

\section{B. Monopoles and their partition function}

Considering the theories on the Coulomb branches, with nonzero VEVs of the scalars $\phi_{A}, A=1 \ldots 6$, one can naturally use the original BPS version of the 't HooftPolyakov monopole, with a mass

$$
M=\left(\frac{4 \pi}{g^{2}}\right) \phi
$$

We will only discuss the $\mathcal{N}=4$ case, following Dorey and collaborators [42]. Six scalars and two holonomies can be combined to vacua parameterized by 8 scalars, extended by supersymmetry to 8 chiral supermultiplets. These 8 fermions have zero modes, describing their binding to monopoles. We will, however, not discuss any of those in detail.

The SU(2) monopole has four collective coordinates, three of which are related with translational symmetry and location in space, while the fourth is rotation around the $\tau^{3}$ color direction,

$$
\hat{\Omega}=\exp \left(i \alpha \hat{\tau}^{3} / 2\right) .
$$

Note that such rotation leaves unchanged the presumed VEVs of the Higgses and holonomies, as well as the Abelian $A_{\mu}^{3} \sim 1 / r$ tails of the monopole solution. Nevertheless, these rotations are meaningful because they do rotate the monopole core-made up of non-Abelian $A_{\mu}^{1}$, $A_{\mu}^{2}$ fields-nontrivially. It is this rotation in the angle $\alpha$ that makes the monopole problem similar to a quantum rotator. As was explained by Julia and Zee [43], the corresponding integer angular momentum is nothing but the electric charge of the rotating monopole, denoted by $q$.

Now that we understand the monopoles and their rotated states, one can define the partition function at certain temperature, which (anticipating the next sections) we will call $T \equiv 1 / \beta$,

$$
\begin{aligned}
Z_{\text {mono }}= & \sum_{k=1}^{\infty} \sum_{q=-\infty}^{\infty}\left(\frac{\beta}{g^{2}}\right)^{8} \frac{k^{11 / 2}}{\beta^{3 / 2} M^{5 / 2}} \\
& \times \exp \left(i k \sigma-i q \omega-\beta k M-\frac{\beta \phi^{2} q^{2}}{2 k M}\right),
\end{aligned}
$$

where $k$ is the magnetic charge of the monopole. The derivation can be found in the original paper, and we only comment that the temperature in the exponent only appears twice, in the denominators of the mass and the rotation terms, as expected. The two other terms in the exponent, $\exp (i k \sigma-i q \omega)$, are the only places where holonomies appear, as the phases picked up by magnetic and electric charges over the circle.

\section{Instantons and monopole-dyons}

Now we derive an alternative four-dimensional version of the theory, in which we will look at gauge field configurations in all coordinates including the compactified "time coordinate" $\tau$. These objects are versions of instantons, split by a nonzero holonomy into instanton constituents. Since these gauge field configurations need to be periodic on the circle, and this condition can be satisfied by paths adding arbitrary number $n$ of rotations, their actions are

$$
S_{\text {mono }}^{n}=\left(\frac{4 \pi}{g^{2}}\right)\left(\beta^{2}|\phi|^{2}+|\omega-2 \pi n|^{2}\right)^{\frac{1}{2}},
$$

including the contribution from the scalar VEV $\phi$, the electric holonomy $\omega$, and the winding number of the path $n$. 
In the absence of the holonomies, the first term would be $M / T$ as one would expect.

The partition function then takes the form [42]

$$
\begin{aligned}
Z_{\text {inst }}= & \sum_{k=1}^{\infty} \sum_{n=-\infty}^{\infty}\left(\frac{\beta}{g^{2}}\right)^{9} \frac{k^{6}}{(\beta M)^{3}} \\
& \times \exp \left(i k \sigma-\beta k M-\frac{k M}{2 \phi^{2} \beta}(\omega-2 \pi n)^{2}\right),
\end{aligned}
$$

where $M=\left(4 \pi \phi / g^{2}\right)$, the BPS monopole mass without holonomies; thus, the second term in the exponent is interpreted as just the Boltzmann factor. The "temperature" appears in the unusual place in the last term (like for the rotator toy model). The actions of the instantons are large at high- $T$ (small circumference $\beta$ ); the semiclassical instanton theory works best at high- $T$.

The Poisson duality relation between these two partition functions, Eqs. (9) and (11), was originally pointed out by Dorey and collaborators [42]. In this paper, it was explained earlier for the simpler toy model of a quantum rotator. Its mathematical origins were presumably clarified enough by our toy model, and it is perhaps enough to remind the reader that the two circles (or the double torus) at play are the angle $\alpha \in[0,2 \pi]$ related with the rotation of the monopole in ordinary/color space and the compactified coordinate $\tau \in[0, \beta]$.

\section{SEMICLASSICAL THEORY AND MONOPOLES IN PURE GAUGE THEORIES}

Now consider theories without adjoint scalars, which do not have an obvious 't Hooft-Polyakov monopole solution. One example of such a theory discussed in Ref. [42] is the $\mathcal{N}=1^{*}$ theory obtained from the $\mathcal{N}=4$ theory by giving a mass to the three chiral multiplets, which, in the IR, eliminates three out of four fermions and all six scalars. We will not discuss this particular case, but proceed directly to pure gauge theory, starting from the instantons.

\section{A. Finite temperature instanton-dyons with an arbitrary time winding}

At zero temperature, the Euclidean space $R^{4}$ is symmetric in all four coordinates, and thus the corresponding saddle points of the integral over fields - the instantonsare four-dimensional spherically symmetric. At finite temperatures, Euclidean time is defined on the circle $\tau \in[0, \beta]$. The corresponding solitons-the calorons-are deformed periodic instantons.

In order to keep the weak coupling and the small density approximation valid, we need to consider sufficiently high $T$. What this means practically will be discussed at the end of the paper. For simplicity, for now we will also ignore the issue of a dynamically generated potential and mean value of the electric holonomy on the time circle, and continue to consider it to be an external parameter; we are therefore considering a "deformed" gauge theory.

The presence of the holonomy is known to split the calorons into $N_{c}$ constituents [29-31] known as instantondyons (or instanton-monopoles). The holonomy eigenvalues $\mu_{i}, i=1 \ldots N_{c}$ enter the gluon and instanton-dyon masses via their differences $\nu_{i}=\mu_{i+1}-\mu_{i}$. We will consider only the simplest case of the number of colors $N_{c}=2$, in which case there is a single holonomy parameter. The caloron is composed of two types of the selfdual dyons, known as the time-independent $M$ dyon and the time-twisted $L$ dyon [44].

Following the discussion above, we need to consider a larger set of saddle-point configurations with all possible periodic paths. To be explicit, let us derive the corresponding semiclassical configurations. One starts with the static BPS monopole, with the $A_{4}$ component of the gauge field now as the adjoint scalar. In the simplest "hedgehog" gauge, the gauge fields are

$$
\begin{aligned}
A_{4}^{a} & =n_{a} v\left(\operatorname{coth}(v r)-\frac{1}{v r}\right), \\
A_{i}^{a} & =\epsilon_{a i j} \frac{n_{j}}{r}\left(1-\frac{v r}{\sinh (v r)}\right),
\end{aligned}
$$

where $n_{a}=x_{a} / r$ is the spatial unit vector and $v$ is the VEV of $A_{4}$ at large distances $r \rightarrow \infty$.

The twisted solution is obtained in two steps. The first is the substitution

$$
v \rightarrow n(2 \pi / \beta)-v,
$$

and the second is the gauge transformation with the gauge matrix,

$$
\hat{\Omega}=\exp \left(-\frac{i}{\beta} n \pi \tau \hat{\sigma}^{3}\right)
$$

where we recall that $\tau=x^{4} \in[0, \beta]$ is the Matsubara time. The derivative term in the gauge transformation adds a constant to $A_{4}$ which cancels out the unwanted $n(2 \pi / \beta)$ term, leaving $v$, the same as for the original static monopole. After "gauge combing" of $v$ into the same direction, this configuration-we will call $L_{n}$-can be combined with any other one. The solutions are all selfdual, but the magnetic and (the Euclidean) electric charges are negative for positive $n$, opposite to the original BPS monopole $M$ for which both are positive. The action corresponding to this solution is

$$
S_{n}=\left(4 \pi / g^{2}\right)|2 \pi n / \beta-v| .
$$

The contribution to the partition function requires the calculation of the pre-exponent, due to quantum 
fluctuations around the $L_{n}$ solution, which have not yet been calculated explicitly.

In the particular case of the $L=L_{1}$ dyon, it can be obtained from the explicit calculation of the moduli for the caloron (finite temperature instanton, the $M+L$ system) in Ref. [44]. For the color SU(2) group, taking the limit of large separation between the dyons, the $L$-dyon contribution has the form

$$
\mathrm{d} Z_{L} \sim \mathrm{d}^{3} x_{L}\left(\frac{8 \pi^{2}}{g^{2}}\right)^{2} e^{-\left(\frac{8 \pi^{2}}{g^{2}}\right) \bar{\nu}}(2 \pi \bar{\nu})^{8 \bar{\nu} / 3}
$$

with $\bar{\nu}=1-\nu$ and $\nu=v T / 2 \pi$. Here the exponent corresponds to the classical action, the power of 2 of the first semiclassical factor stems from the zero modes $\frac{1}{2} N_{\text {zero modes }}$, and the last factor stems from the large distance limit of the volume of the moduli space according to [44], see also Appendix C of Ref. [32].

Unlike in the theories with extended supersymmetry, in QCD-like theories there are no (partial) cancellations in the determinant of the nonzero modes between bosons and fermions, and the normalization constant require tedious calculation of the nonzero mode part which was not yet done. The renormalizability however requires that it appends the bare charge in the action to the correct running coupling at the relevant scale is reproduced. This means that one expects the exponent to read

$$
\begin{aligned}
\mathrm{d}_{L_{n}} & \sim \mathrm{d}^{3} x_{L} \\
& \times \exp \left[-|2 \pi T n-v|\left(\frac{8 \pi^{2}}{g_{0}^{2}}+\frac{22}{3} \log \left(\frac{p_{0}}{|2 \pi T n-v|}\right)\right)\right],
\end{aligned}
$$

where the coupling $g_{0}$ is defined at the normalization scale $p_{0}$. Similarly, the power of the action in the numerator must be appended by the two-loop corrections to the two-loop beta function, and so on.

For our subsequent discussion, we will ignore the running and only keep the first term, taking the mean coupling to be just a constant at a characteristic $p_{0}=2 \pi T\langle\bar{\nu}\rangle$, say

$$
S_{0} \equiv S_{L}+S_{M}=\frac{8 \pi^{2}}{g_{0}^{2}}=10 .
$$

The simulation of instanton-dyon ensembles [32] were done for $S_{0}$ ranging from 5 to 13 , and thus defining a rather large range of dyon densities. Higher-twist instantons $L_{n}$ for $n>1$ or $n<0$ are all strongly suppressed and in practice can be ignored; the instanton-dyon ensemble calculations performed in Ref. [32] only included the $n=0$ time independent dyon $M$ and the first twisted dyons $L_{1}$ because, in this range of temperatures, the holonomy phase $\omega$ changes from a small value to $\pi$ at the confining phase transition, where $\omega$ and $2 \pi-\omega$ are comparable.

In the present calculation, we will keep all of them, preserving exact periodicity, and write the semiclassical partition function as

$$
Z_{\text {inst }}=\sum_{n} e^{-\left(\frac{4 \pi}{g_{0}^{2}}\right)|2 \pi n-\omega|}
$$

It is periodic in the holonomy, as it should be. Note that, unlike in Eq. (11), it has a modulus rather than a square of the corresponding expression in the exponent. This is due to the fact that the sizes of $L_{n}$ and their masses are all defined by the same combination $|2 \pi n-\omega| T$ and therefore the moment of inertia $\Lambda \sim 1 /|2 \pi n \beta-v|$.

\section{B. The Poisson transformation}

A key point of this paper is that the existence of the semiclassical instanton partition function implies the existence of monopoles moving and rotating in their collective coordinates. According to the general Poisson relation, Eq. (6), the Fourier transform of the corresponding function appearing in the sum in Eq. (19) reads

$$
\begin{aligned}
F\left(e^{-A|x|}\right) & \equiv \int_{-\infty}^{\infty} \mathrm{d} x e^{i 2 \pi \nu x-A|x|} \\
& =\frac{2 A}{A^{2}+(2 \pi \nu)^{2}},
\end{aligned}
$$

and therefore the monopole partition function is

$$
Z_{\mathrm{mono}} \sim \sum_{q=-\infty}^{\infty} e^{i q \omega-S(q)},
$$

where

$$
\begin{aligned}
S(q) & =\log \left(\left(\frac{4 \pi^{2}}{g_{0}^{2}}\right)^{2}+q^{2}\right) \\
& \approx 2 \log \left(\frac{4 \pi^{2}}{g_{0}^{2}}\right)+q^{2}\left(\frac{g_{0}^{2}}{4 \pi}\right)^{2}+\ldots,
\end{aligned}
$$

where the last equality is for $q \ll 4 \pi^{2} / g_{0}^{2}$.

\section{WHAT HAVE WE LEARNED ABOUT THE QCD MONOPOLES?}

Before summarizing our answer to this question, let us first recall the setting and conclusions of the preceding section. The coupling is presumed small, so the action at the relevant scale is large $4 \pi / g^{2} \gg 1$, and the semiclassical calculation is well controlled. This implies that the corresponding temperature is "high enough." The holonomies $\omega, \sigma$, treated as external Aharonov-Bohm phases imposed on the system, create a certain "Higgsing" of the gluons, 
with only the diagonal ones remaining massless. Calorons are split into the instanton-dyons, and the semiclassical partition function, appended by all $L_{n}$ contributions, can be calculated.

What we would actually like to study is the QCD, with quarks, at temperatures around the deconfinement transition $T \sim T_{c}$. Indeed, heavy-ion collisions create matter with $T$ between roughly $2 T_{c} \approx 300 \mathrm{MeV}$ and $0.5 T_{c}$. Most finite- $T$ lattice studies are devoted to this temperature range as well. In this regime the action of the $L_{1}$ instanton-dyon is numerically

$$
S_{L_{1}}=\frac{4 \pi^{2}}{g^{2}} \sim 4
$$

with $L_{n}$ having larger actions respectively. So, the semiclassical suppression $\exp \left(-S_{L_{1}}\right) \approx e^{-4}$ seems to be robust enough to keep only $L_{1}$ and $M$ dyons. But, including the preexponents, one finds that the ensemble is not really dilute, and in order to perform the integration over the collective variables, one needs to solve a nontrivial manybody problem of a dense instanton-dyon plasma. The instanton-dyon ensemble of $M, L_{1}$ dyons does shift the potential for the electric holonomy dynamically to its "confining" value, for $T<T_{c}$. Semiclassical ensembles of instanton-dyons also explain chiral symmetry breaking, and their changes with flavor-dependent quark periodicity phases. Further development of the semiclassical theory is, therefore, well justified.

The main point of this paper is that any semiclassical partition function, once derived, can be Poisson-rewritten into an identical " $\mathrm{H}$ " form, with the sum over certain excited states. We have shown how one can do so for pure gauge theory, without scalars, using (relatively schematic form of) the semiclassical partition function, for which we calculated its Poisson dual. We further argued that the resulting partition function can be interpreted as being generated by moving and rotating monopoles.

The results are a bit surprising. First, the action of a monopole, although still formally large in weak coupling, is only a logarithm of the semiclassical parameter; these monopoles are therefore lighter than the instanton-dyons. While the specific value of the monopole action,

$$
S_{\text {mono }}=2 \log \left(\frac{4 \pi^{2}}{g^{2}}\right) \approx 2 \log (4 .) \approx 2.8,
$$

can still be considered larger than 1 , one may ask whether it can be even parametrically modified by the powers of logs in the instanton partition function. In summary, it is not yet clear whether those monopoles are or are not truly semiclassical.

Second is the issue of monopole rotation. The very presence of an object that admits rotational states implies that the monopole core is not spherically symmetric.
The Poisson-rewritten partition function has demonstrated that the rotating monopoles are not rigid rotators, because their action, Eq. (22), depends on the angular momentum $q$ and is quadratic only for small values of $q$. The slow (logarithmic) increase of the action with $q$ implies that the dyons are in fact shrinking with increased rotation. In the moment of inertia, this shrinkage is more important than the growth in the mass, as the size appears quadratically. As strange as it sounds, it reflects on the corresponding behavior of the instanton-dyons $L_{n}$ with the increasing $n$.

Although such rotations are well known in principle as Julia-Zee dyons with real electric charge (unlike that of the instanton-dyons, which only exist in the Euclidean world) and studied in theories with extended supersymmetries, to our knowledge the existence of multiple rotational states of monopoles has not yet been explored in monopole-based phenomenology. In particular, one may wonder how the existence of multiple rotational states affects their Bosecondensation at $T<T_{c}$, the basic mechanism behind the deconfinement transition. The electric charges of the rotating monopoles should, therefore, also contribute to the jet quenching parameter $\hat{q}$ and the viscosity, which was not included before.

We note that perhaps a useful "middle ground" connecting the two regimes - the idealized semiclassical dilute gas and the real-life finite- $T$ QCD—would be lattice studies of the gauge theory with an induced holonomy at high $T$. To our knowledge, this has not been done in detail.

The semiclassical studies in this direction by introduction of certain masses, by Dorey et al. using $\mathcal{N}=4 \rightarrow$ $\mathcal{N}=1$ [42] and by Unsal et al. for $\mathcal{N}=2$ deformed toward pure gauge theory [38], were the first steps in this direction. Yet the conjectured continuity of both the confined and deconfined phases, from dilute to dense regimes, were never studied nor confirmed.

Lattice measurements of the holonomy potential for the $\mathrm{SU}(2)$ and $\mathrm{SU}(3)$ gauge theories with fixed external holonomy have been performed in Ref. [45]. The perturbative renormalized potential was derived and compared to these data in Ref. [46]. Such a subtraction opens the door to studies of the monopole contribution, which has not been attempted as of yet. Other lattice studies, of the "deformed QCD" with an extra holonomydependent term in the action, were performed in Ref. [47]. Finally, the introduction of light quarks allows the study of zero modes of both semiclassical instantondyons and monopoles, suggesting another way to test their mutual correspondence.

\section{ACKNOWLEDGMENTS}

This work was supported by the U.S. Department of Energy, Office of Science under Contract No. DE-FG88ER40388. We also thank G. Dunne, who pointed out to us the early literature on the idea of the Poisson duality. 
Note added.-After the paper was finished and submitted, we learned that some features of the "rotator,"used in Sec. II as the simplest example of the Poisson duality, have been considered before in the literature on path integrals over compact manifolds and Lie groups. Specifically, the result (5) for both partition functions in terms of the $\theta_{3}$ Jacobi theta function has been derived already in a classic paper by L. Schulman [48], who also noticed that the Jacobi identity needed to prove their equality, $\theta_{3}(z, t)=(-i t)^{-1 / 2} e^{z^{2} / i \pi t} \theta_{3}(z / t,-1 / t)$, can be derived using the Poisson summation formula, the fact which we emphasized.
[1] P. A. M. Dirac, Proc. R. Soc. A 133, 60 (1931).

[2] G. 't Hooft, Nucl. Phys. B79, 276 (1974).

[3] A. M. Polyakov, Pis'ma Zh. Eksp. Teor. Fiz. 20, 194 (1974) JETP Lett. 20, 430 (1974).

[4] N. Seiberg and E. Witten, Nucl. Phys. B426, 19 (1994); Nucl. Phys. B430, 485(E) (1994).

[5] Y. Nambu, Phys. Rev. D 10, 4262 (1974).

[6] G. 't Hooft, Nucl. Phys. B190, 455 (1981).

[7] S. Mandelstam, Phys. Rep. 23, 245 (1976).

[8] M. L. Laursen and G. Schierholz, Z. Phys. C 38, 501 (1988).

[9] Y. Koma, M. Koma, E. M. Ilgenfritz, T. Suzuki, and M. I. Polikarpov, Phys. Rev. D 68, 094018 (2003).

[10] V. G. Bornyakov et al. (DIK Collaboration), Phys. Rev. D 70, 074511 (2004).

[11] T. Suzuki, M. Hasegawa, K. Ishiguro, Y. Koma, and T. Sekido, Phys. Rev. D 80, 054504 (2009).

[12] A. D'Alessandro and M. D’Elia, Nucl. Phys. B799, 241 (2008).

[13] C. Bonati and M. D’Elia, Nucl. Phys. B877, 233 (2013).

[14] J. Liao and E. Shuryak, Phys. Rev. Lett. 101, 162302 (2008).

[15] A. D’Alessandro, M. D'Elia, and E. V. Shuryak, Phys. Rev. D 81, 094501 (2010).

[16] A. Ramamurti and E. Shuryak, Phys. Rev. D 95, 076019 (2017).

[17] C. Bonati, A. Di Giacomo, L. Lepori, and F. Pucci, Phys. Rev. D 81, 085022 (2010).

[18] C. Bonati, A. Di Giacomo, and M. D’Elia, Phys. Rev. D 82, 094509 (2010).

[19] A. Di Giacomo, arXiv:1707.07896.

[20] J. Liao and E. Shuryak, Phys. Rev. C 75, 054907 (2007).

[21] J. Liao and E. Shuryak, Phys. Rev. C 77, 064905 (2008).

[22] A. Ramamurti and E. Shuryak, Phys. Rev. D 97, 016010 (2018).

[23] C. Ratti and E. Shuryak, Phys. Rev. D 80, 034004 (2009).

[24] J. Xu, J. Liao, and M. Gyulassy, J. High Energy Phys. 02 (2016) 169.
[25] J. Xu, J. Liao, and M. Gyulassy, Chin. Phys. Lett. 32, 092501 (2015).

[26] P. Cea, L. Cosmai, F. Cuteri, and A. Papa, Phys. Rev. D 95, 114511 (2017); EPJ Web Conf. 175, 12006 (2018).

[27] A. Ramamurti and E. Shuryak, arXiv:1801.06922.

[28] A. A. Belavin, A. M. Polyakov, A. S. Schwartz, and Y. S. Tyupkin, Phys. Lett. 59B, 85 (1975).

[29] T. C. Kraan and P. van Baal, Phys. Lett. B 428, 268 (1998).

[30] T. C. Kraan and P. van Baal, Nucl. Phys. B533, 627 (1998).

[31] K. M. Lee and C. h. Lu, Phys. Rev. D 58, 025011 (1998).

[32] R. Larsen and E. Shuryak, Phys. Rev. D 92, 094022 (2015).

[33] R. Larsen and E. Shuryak, Phys. Rev. D 93, 054029 (2016).

[34] Y. Liu, E. Shuryak, and I. Zahed, Phys. Rev. D 92, 085006 (2015).

[35] Y. Liu, E. Shuryak, and I. Zahed, Phys. Rev. D 92, 085007 (2015).

[36] E. Shuryak, EPJ Web Conf. 175, 12001 (2018).

[37] N. Dorey and A. Parnachev, J. High Energy Phys. 08 (2001) 059.

[38] E. Poppitz and M. Unsal, J. High Energy Phys. 07 (2011) 082.

[39] E. Poppitz, T. Schfer, and M. Unsal, J. High Energy Phys. 10 (2012) 115.

[40] Y. Aharonov and D. Bohm, Phys. Rev. 115, 485 (1959).

[41] A. Zygmund, Trigonometric Series, 2nd ed. (Cambridge University Press, Cambridge, England, 1968).

[42] N. Dorey, J. High Energy Phys. 04 (2001) 008.

[43] B. Julia and A. Zee, Phys. Rev. D 11, 2227 (1975).

[44] D. Diakonov, N. Gromov, V. Petrov, and S. Slizovskiy, Phys. Rev. D 70, 036003 (2004).

[45] D. Diakonov, C. Gattringer, and H. P. Schadler, J. High Energy Phys. 08 (2012) 128.

[46] D. Diakonov, V. Petrov, H. P. Schadler, and C. Gattringer, J. High Energy Phys. 11 (2013) 207.

[47] M. Ogilvie and P. Meisinger, Proc. Sci., LATTICE2014 (2014) 339, arXiv:1411.5344.

[48] L. Schulman, Phys. Rev. 176, 1558 (1968). 